\title{
Cryo-Electron Tomography of Malaria Parasites
}

\author{
M. Cyrklaff, M. Kudryashev, N. Kilian, P. Henrich, F. Frischknecht and M. Lanzer \\ Hygiene Institut, Abteilung Parasitologie, Universitätsklinikum Heidelberg, Im Neuenheimer Feld \\ 324, 69120 Heidelberg, Germany
}

Cryo-electron tomography (cryo-ET) has recently emerged as a method of choice for studying structures of complex biological assemblies as well as the morphology of intact cells. Biological material is preserved in a native-hydrated state by rapid freezing and the resulting 3D maps enable detailed structural characterization [1].

We use cryo-ET to study structure-function relationship of the Plasmodium parasites, the causative agents of malaria. In a broad approach we aim on different stages of the parasite life cycle. The mobile sporozoites, small (0.5-1 micron thick), highly polarized cells that are generated in a mosquito vector and after transmission to a vertebrate host move towards and invade the hepatocytes, are deposited on EM grids, plunge frozen and studied en toto. Resulting tomograms (Fig. 1) revealed unexpected organization of microtubule cytoskeleton [2]. Further analysis revealed the presence of proteinous material organized in a network of fibers and linkers that interconnect cytoskeleton, plasma membrane, inner membrane complex, as well as various organelles in a cell. This material may also endow a characteristic, polarized shape of the sporozoites.

In the further approach we aim on investigating blood stages of Plasmodium falciparum. The project involves scoping into a complex between two organisms, the pathogen and the host erythrocyte interacting with each other. Of a special interest are the structures assembled by the parasite in the cytoplasm of host erythrocyte, i.e. beyond the cellular constrains of the parasite. These are implicated in the export of parasite antigens to the cell surface [3], which then induce an aggregation of infected erythrocytes in microvasculature, leading to severe symptoms of the cerebral and placental malaria. Central to such export are membranous structures, referred to as Maurer's clefts, as well as the networks of tubes and vesicles [3]. The tomography on the whole-cell cryopreparations (Fig. 2) as well as on the sections of both cryo-hydrated and freeze-substituted material revealed remarkable details of the parasitic export and allowed detailed analysis of the structures preserved in the native state. In particular, resolving the interconnections between subsequent compartments gives an explanation to the nature of export pathways, which in turn will advance the studies on the export of parasitic factors to cell surface, and potentially to develop strategies of how to impede the export and cell adhesion. Additionally, by using EM-immuno methods in cryopreserved samples we localized the essential antigens of the parasitic export systems in correlation with the intact structures [4].

References:

[1] V. Lucić et al., Ann. Rev. Biochem. 74 (2005) 833.

[2] M. Cyrklaff et al., J. Exp. Med. 204 (2007) 1281.

[3] M. Lanzer et al., Int. J. Parasitol. 36 (2006) 23.

[4] P. Henrich et al., (2009) submitted. 


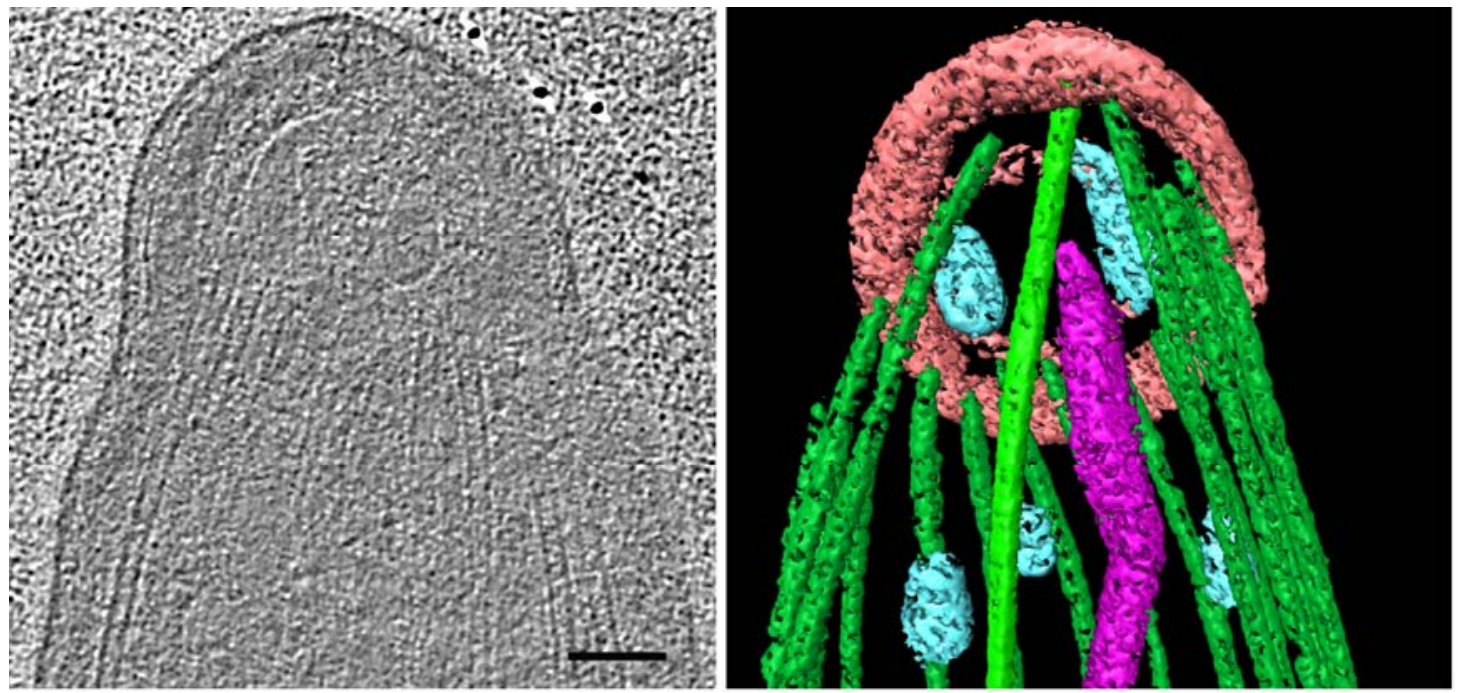

Fig. 1. Cryo-tomographic reconstruction of the apical region of frozen-hydrated Plasmodium berghei sporozoite, the causative agents of malaria; left - a $12 \mathrm{~nm}$ thick section through the tomogram; right - surface rendered view of the same area showing selected features: microtubules (green), polar rings - microtubule organising centres (pink), rhoptry (turquoise) micronemes (violet). Scale bar $=100$ nm.

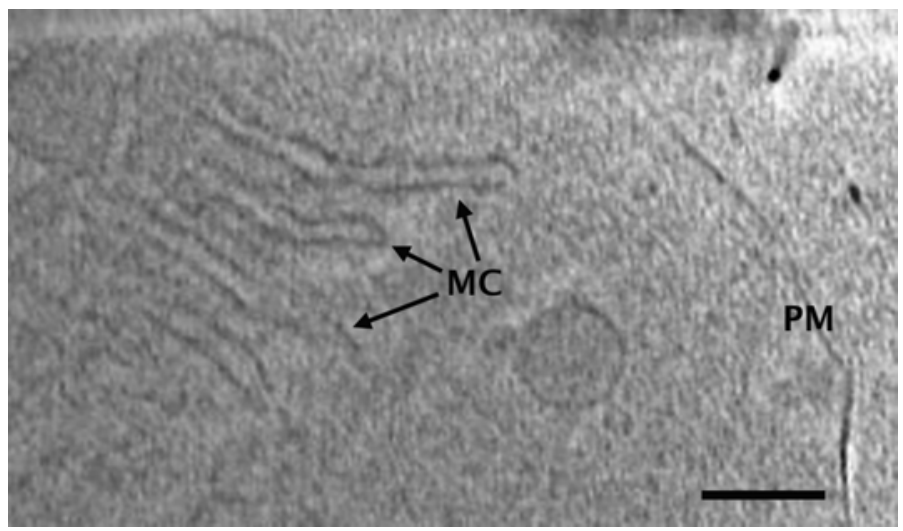

Fig. 2. A slice through the cryo-electron tomogram of an erythrocyte infected with Plasmodium falciparum, showing cisternae of a Maurer's cleft $(\mathrm{MC}) ;(\mathrm{PM}=$ plasma membrane $)$. Scale bar $=100$ nm. 